A N N A L E S Annales de Bretagne et des Pays de l'Ouest

\title{
Alain Valais, L'habitat rural au Moyen Âge dans le Nord-Ouest de la France
}

Daniel Pichot

\section{(2) OpenEdition}

1 Journals

\section{Édition électronique}

URL : http://journals.openedition.org/abpo/2884

DOI : $10.4000 / a b p o .2884$

ISBN : 978-2-7535-3977-8

ISSN : 2108-6443

\section{Éditeur}

Presses universitaires de Rennes

Édition imprimée

Date de publication : 15 décembre 2014

Pagination : 179-181

ISBN : 978-2-7535-3975-4

ISSN : 0399-0826

\section{Référence électronique}

Daniel Pichot, « Alain Valais, L'habitat rural au Moyen Âge dans le Nord-Ouest de la France », Annales de Bretagne et des Pays de l'Ouest [En ligne], 121-4 | 2014, mis en ligne le 15 décembre 2014, consulté le 22 septembre 2020. URL : http://journals.openedition.org/abpo/2884 ; DOI : https://doi.org/10.4000/ abpo. 2884 


\section{Comptes rendus}

VALAIS, Alain (dir.), L'habitat rural au Moyen Âge dans le Nord-Ouest de la France, t. 1, Les synthèses, 328 p, t. 2 : Les notices. Préface de Elizabeth Zadora-Rio, Rennes, PUR, 2012, 462 p. ISBN : 978-2-7535-1780-6, $35 €$

Au terme d'une longue enquête, et malheureusement de nombreuses années d'attente d'un éditeur, Alain Valais nous livre une magnifique synthèse sur l'habitat rural en Anjou à l'époque médiévale. Il faut saluer l'importance de la synthèse et le travail d'édition particulièrement soigné, cartes et croquis nombreux bénéficiant d'une impression de grande qualité et dans des dimensions qui en facilitent la lecture et l'utilisation. Ce gros travail dont les deux volumes totalisent presque 800 pages grand format apporte une contribution majeure à nos connaissances sur l'habitat rural médiéval et cela, dans le cadre d'une vaste synthèse, pratique que l'on souhaiterait plus fréquente dans l'enquête archéologique. Nous est présenté le fruit d'un Projet Collectif de Recherche qui a fonctionné entre 1990 et 2000 en essayant de rassembler la documentation sur le sujet et d'en dresser une série d'études synthétiques, le point de départ étant les nombreux sites découverts pendant les travaux autoroutiers qui ont traversé la Sarthe et le Maine-et-Loire, A84, 85, 87. Le domaine de l'habitat rural, fort peu documenté jusqu'ici au regard d'autres secteurs : nécropoles, châteaux, bénéficie ainsi d'une remarquable mise en lumière.

Il faut commencer par le tome II, celui qui regroupe les 50 notices des différents sites étudiés. Les auteurs y ont rassemblé tous les éléments relatifs à des chantiers de fouilles en milieu rural, la plupart liés aux constructions autoroutières mais en ajoutant des travaux plus anciens, certains jamais publiés. Le plus grand nombre concerne le Maine-et-Loire mais les départements périphériques apportent d'utiles compléments et même l'Ille-et-Vilaine avec un seul site, certes, met en évidence la transgression de la frontière régionale. Un gros travail a été fourni pour normaliser ces notices afin qu'elles présentent, autant que possible, un ensemble homogène autorisant travail statistique et comparaison. La chose était compliquée en raison de l'étalement dans le temps des chantiers et de la diversité des pratiques et des choix. Après les renseignements techniques, viennent le résumé, une présentation du contexte géographique et archéologique, et enfin, les éléments trouvés en fouilles et une interprétation. Une bibliographie clôt l'ensemble. Pour certaines fiches intervient une annexe, en particulier les inventaires de mobilier, céramiques ou objets métalliques, plusieurs cas présentent un très grand intérêt, en particulier pour établir des catalogues et obtenir des références chronologiques.

Bien entendu, le développement de ces notices présente une très grande inégalité en fonction de l'importance du site ou de ce qui a subsisté de fouilles anciennes. La fiche 1, celle de la Tullaye en Janzé (35) illustre bien le problème : 6 pages seulement pour un grand décapage présentant l'évolution d'un site d'habitat à propos duquel n'avaient naguère été publiées que de très brèves notes. À l'inverse, Les Murailles, longuement fouillées par A. Valais se voient consacrer un nombre important de pages, la qualité du site et les renseignements qu'il fournit le justifiant amplement. Pour le reste, s'égrènent des sites variés mais où domine assez nettement le 
haut Moyen Âge avec ses trous de poteaux et ses réseaux fossoyés. S'y ajoutent, pour certains, des activités artisanales : textiles, sidérurgie, céramique. Enfin une fouille vendéenne met en valeur une série de pirogues. Souvent, les fiches trahissent la grande modestie du chantier mais c'est bien l'intérêt du travail, le rapprochement qui, en créant un effet de nombre, sinon de masse, donne de l'intérêt au moindre site. Ce volume est une incontestable réussite et, en rendant accessible nombre de rapports de fouilles, il offre une documentation majeure et plus facilement utilisable en raison de sa mise aux normes.

À partir de ce recueil, un certain nombre de chercheurs se sont chargés de dégager neuf synthèses qui mettent en valeur les données acquises dans l'inventaire et offrent un notable apport à l'étude de l'habitat médiéval, essentiellement sous ses formes dispersées dont l'intérêt est largement souligné dans la préface d'E. ZadoraRio. Les deux premiers rapports s'intéressent à l'organisation spatiale des sites et aux constructions. Dans un développement très dense et long de 60 pages, Frédéric Guérin cerne les organisations spatiales et leur évolution. Il souligne l'importance du substrat ancien présent une fois sur deux mais sans entrainer une permanence fonctionnelle. Le site reste mais évolue vers d'autres formes. Une phase de créations importante se place aux $\mathrm{VI}^{\mathrm{e}}$-VII ${ }^{\mathrm{e}}$ siècle et une autre, moindre, à l'époque carolingienne, marquée par une certaine diversité. Le site privilégié et aristocratique de Distré, Les Murailles, domine largement La Tullaye ou Saint-Sylvain d'Anjou et encore plus bien des sites fort modestes. À cette époque l'activité artisanale domestique est plus nette : métallurgie et textile surtout mais teinture à Pornic. Les sépultures, généralement groupées, demeurent bien présentes en périphérie de l'habitat mais un véritable cimetière existe à Sainte-Hermine. Le Moyen Âge classique apparaît plus discret mais semble surtout marqué par une réorganisation que traduit un fort mouvement d'abandon qui culmine au xII ${ }^{\mathrm{e}}$ siècle. Alain Valais analyse ensuite plus brièvement les techniques de construction. Il met particulièrement en valeur un habitat marqué par le bois. Les bâtiments excavés mérovingiens sont bien attestés dans l'Ouest mais leur fonction d'annexe demeure quand même à approfondir. La période carolingienne apparaît mieux, les annexes excavées subsistent mais dominent les constructions sur poteaux. À Distré les demeures ne possédant généralement qu'une pièce sont bâties sur des solins de pierre et s'accompagnent de silos et de greniers sur poteaux.

La vie quotidienne étudiée par Christophe Devals aborde les domaines du vêtement, des lampes, des jeux, de l'artisanat. On retiendra surtout le long développement consacré aux zones dédiées aux différents types de silos et à leur fonction. Les chapitres suivants sont réservés à des aspects spécifiques particulièrement riches. L'étude et l'inventaire des objets métalliques de Distré mettent en lumière l'importance du site, dégageant armes et outils (Vincent Legros) tandis que Isabelle MoreraVincotte se consacre à un essai de synthèse des céramiques régionales de grande importance dans lequel beaucoup de chercheurs seront amenés à puiser. La mise au point d'une typologie permet aussi de rendre compte des habitudes culinaires qui subissent une forte évolution à la fin du Moyen Âge. Enfin deux courts articles sont consacrés à des aspects majeurs de la nourriture. Les débris de cuisine et les déchets permettent une approche de l'alimentation carnée tandis que les meules éclairent sur la transformation des céréales. Logiquement, la région présente de fortes analogies avec la France du nord mais se distingue aussi par des caractères particuliers, aristocratiques, pour le site des Murailles.

Les auteurs ont enfin eu l'idée de demander à l'iconographie un complément d'information. La région bien pourvue de peintures murales a permis l'élaboration d'un corpus qui complète et éclaire les résultats de la fouille. Les outils y apparaissent en entier et surtout dans leur utilisation. Tout en tenant compte des biais 
que l'image médiévale impose, il faut reconnaître l'intérêt de cette initiative dont le résultat est indéniable.

Enfin, Anne Nissen-Jaubert élargit le débat. Elle replace cette étude de l'Ouest dans les travaux actuels sur l'Occident et aborde la question de la continuité et/ ou rupture en posant le problème autrement et avec beaucoup de nuances. Les données chronologiques souvent larges ne permettent pas de fixer les différentes phases avec précision mais l'auteur relève le maintien des éléments structurants comme les chemins et les limites de parcelles, ce qui n'exclut pas une forte mobilité de l'habitat.

Le compte-rendu ne peut entrer dans les détails et montrer tout ce que le spécialiste des études régionales pourra trouver en termes de données et de comparaisons. Incontestablement, ces deux volumes sont une réussite. On hésite à émettre des regrets mais, puisque ce vaste corpus aborde la question de l'habitat, pourquoi ne pas entrer plus en débat avec les historiens qui se sont penchés sur la question? Quelques lignes de la conclusion nous laissent sur notre faim. En 2000, date d'achèvement de l'enquête, des travaux étaient déjà publiées, en particulier le volume de Flaran consacré à l'habitat dispersé. Les sites de l'étude appartiennent très majoritairement à cette catégorie d'habitats qui, bien que nommés écarts, terme un peu péjoratif, constituent l'élément majeur car le plus fréquent de la résidence des hommes, bien plus que le centre ecclésial qui ne regroupe souvent qu'un nombre limité d'habitants. Cette question essentielle ne peut progresser que par un débat nécessaire. Il faut le reconnaître, un tel travail rend ce dialogue possible en offrant un corpus de données et une réflexion de grande qualité, et souhaitable, car il devrait produire des fruits du plus grand intérêt.

Daniel PICHOT

FAVReau, Robert (dir.), Poitiers, de Jean de Berry à Charles VII. Registres de délibérations du corps de ville $n^{\circ} 1,2$ et 3 (1412-1448), Archives historiques du Poitou, t. LXVI, 2014, 464 p.

La collection des Archives historiques du Poitou vient de s'enrichir d'un $66^{\mathrm{e}}$ volume avec la publication des trois plus anciens registres de délibérations du corps de ville de Poitiers conservés pour les années 1412-1448. C'est le premier tome d'une série qui en comptera quatre à terme et qui couvrira l'édition intégrale des sept registres de délibérations municipales du Xve siècle aujourd'hui conservés à la médiathèque de Poitiers.

Une courte introduction (p. 7-14) présente ces sept registres, dont les trois qui font l'objet de la présente édition : le ${ }^{\circ} 1$, années 1412-1420; le $\mathrm{n}^{\circ} 2$, années 14211428 et le $n^{\circ} 3$, années 1436-1448. Une brève mise en perspective avec les autres publications de sources municipales pour les villes françaises montre combien le genre était tombé en déshérence de longue date.

Couvrant, malgré des lacunes, une grande partie de la première moitié du $\mathrm{XV}^{\mathrm{e}}$ siècle, ces trois registres permettent à l'historien de plonger au cœur des derniers soubresauts de la guerre de Cent Ans, à l'ombre de deux personnages marquants avec lesquels Poitiers a entretenu des relations plus ou moins étroites : Jean de Berry, flamboyant prince apanagiste détenteur du comté de Poitiers (mort en 1416, auquel son fils, également prénommé Jean, ne survécut qu'un an, le comté faisant alors retour à la Couronne), puis le dauphin Charles - «monsieur le Régent " comme se plaisent à l'appeler les sources poitevines - avant et après sa mue politique en roi 